\section{Aripiprazole Treatment for Coprophagia in Autistic Disorder}

To the Editor: Coprophagia (ingestion of feces) is considered a variant of pica that can generate intense emotional reactions in those exposed to it. To date, its psychopathological bases remain ill-understood, and clear evidence for its treatment is lacking.

We report a case of coprophagia occurring in a young man with autistic spectrum disorder that subsided after a short treatment with aripiprazole, an atypical antipsychotic with $\mathrm{D}_{2}$ partial agonist properties, 5HT2A antagonist actions, and 5HT1A partial agonist actions.

\section{Case Report}

Our patient is a 29-year-old man with high-functioning autistic spectrum disorder, diagnosed by experienced clinicians and confirmed by validated scales. While severely impaired in spoken expression, the patient presented with some written communication skills and was able to complete high school. In his usual state of health, the patient presented with motor stereotypies and significant impairment in social interactions, consistent with his diagnosis. History was negative for difficulties with toilet training or significant bowel disease. The patient developed coprophagia with a daily frequency while under treatment with risperidone, $0.5 \mathrm{mg}$ b.i.d. At the same time, the patient also showed an increase in aberrant motor behaviors. Blood studies, including thiamine concentration, and neurological examination were unrevealing. Mental status examination was unchanged, and it was negative for thought or perceptual disturbances.

After 2 weeks without any behavioral changes, treatment with risperidone was discontinued, and aripiprazole, $15 \mathrm{mg} /$ day, was prescribed to the patient. After 4 weeks of aripiprazole treatment, the patient presented a marked reduction in the coprophagic behaviors, which receded substantially after 8 weeks of treatment. The drug was well tolerated with no side effects.

\section{Discussion}

Coprophagia has been linked with different conditions including melancholia, schizophrenia, mental retardation, and organic brain disorders ${ }^{1}$ while its occurrence in high-functioning autism has not, to our knowledge, been reported before. Different treatments for coprophagia are reported, including behavioral therapy, dietary changes, tricyclic antidepressants, haloperidol, perospirone, SSRIs, and ECT, all of them showing partial efficacy. ${ }^{1,2}$ However, as pharmacological treatments in autistic spectrum disorders are known to be challenging due to hypersensitivity and intolerance to side effects, some of the therapeutic protocols reported in the literature could be unsuitable for these patients. Our case seems to suggest that aripiprazole presents with the potential to control complex behavioral manifestations such as coprophagia in patients with autistic spectrum disorders.

Matteo Pardini, M.D.

Silvia Guida, M.D.

Department of Neurosciences, Ophthalmology and Genetics, University of Genoa, Genoa, Italy

Leonardo Emberti Gialloreti, M.D., PH.D.

Department of Public Health and Cell Biology, Tor Vergata University, Rome

\section{References}

1. Beck DA, Frohberg NR: Coprophagia in an elderly man: a case report and review of the literature. Int J Psychiatry Med 2005; 35:417-427

2. Harada KI, Yamamoto K, Saito T: Effective treatment of coprophagia in a patient with schizophrenia with the novel atypical antipsychotic drug perospirone. Pharmacopsychiatry 2006; 39:113 